\title{
Role of the lattice in the light-induced insulator-to-metal transition in vanadium dioxide
}

\author{
Cédric Weber, ${ }^{1, *}$ Swagata Acharya $\odot,{ }^{1}$ Brian Cunningham $\odot,{ }^{2}$ Myrta Grüning, ${ }^{2}$ Liangliang Zhang, ${ }^{3}$ Hang Zhao, ${ }^{4}$ Yong Tan ${ }^{4}$ \\ Yan Zhang $\odot,{ }^{3}$ Cunlin Zhang, ${ }^{3}$ Kai Liu, ${ }^{5}$ Mark Van Schilfgaarde,,${ }^{1,6}$ and Mostafa Shalaby ${ }^{3,7, \dagger}$ \\ ${ }^{1}$ King's College London, The Strand, WC2R 2LS London, United Kingdom \\ ${ }^{2}$ School of Mathematics and Physics, Queen's University Belfast, Belfast BT7 1NN, United Kingdom \\ ${ }^{3}$ Key Laboratory of Terahertz, Optoelectronics, Beijing Advanced, Innovation Center for Imaging Technology CNU, Beijing 100048, China \\ ${ }^{4}$ Beijing Key Laboratory for Precision Optoelectronic Measurement Instrument and Technology, School of Optics and Photonics, \\ Beijing Institute of Technology, Beijing 100081, China \\ ${ }^{5}$ State Key Laboratory of New Ceramics and Fine Processing, School of Materials Science and Engineering, Tsinghua University, \\ Beijing 100084, China \\ ${ }^{6}$ National Renewable Energy Laboratories, Golden, Colorado 80401, USA \\ ${ }^{7}$ Swiss Terahertz Research-Zurich, Technopark, 8005 Zurich, Switzerland and Park Innovaare, 5234 Villigen, Switzerland
}

(Received 18 September 2019; revised manuscript received 16 December 2019; accepted 19 February 2020; published 24 April 2020)

\begin{abstract}
Vanadium dioxide is a model material system which exhibits a metal-to-insulator transition at $67^{\circ} \mathrm{C}$. This holds potential for future ultrafast switching in memory devices but typically requires a purely electronic process to avoid the slow lattice response. The role of lattice vibrations is thus important, but it is not fully understood and it has been a long-standing source of controversy. We use ultrafast spectroscopy and $a b$ initio quantum calculations to study the mechanism responsible for the transition. We identify an atypical Peierls vibrational mode associated with the transition and study its associated properties. Our findings led to theoretical prediction of a new electron-phonon pathway for a purely reversible electronic transition in a true bistable fashion under specific conditions. This transition is very atypical, as it involves purely chargelike excitations and requires only small nuclear displacement and might prompt future experimental investigations.
\end{abstract}

DOI: 10.1103/PhysRevResearch.2.023076

\section{INTRODUCTION}

Correlated electron systems offer various pathways of controlling material properties. For example, vanadium dioxide $\left(\mathrm{VO}_{2}\right)$ is an insulator with strong electron-phonon interactions and it undergoes a first-order insulator-to-metal transition (IMT) at $340 \mathrm{~K}$ [1-4]. At higher temperatures, $\mathrm{VO}_{2}$ is metallic with the rutile structure $(\mathrm{R})$, while it transforms to the monoclinic $\mathrm{M}_{1}$ phase and becomes insulating below the transition temperature. IMT can be achieved on the ultrafast timescale on laser excitation [5-8]. However, bistability is normally not reached as the system undergoes a hysteresis. Whether the transition involves both electrons and phonons or only electrons remains a topic of high controversy. The involvement of the lattice is detrimental for ultrafast switching, as it is typically associated with slow response and high power consumption. In this context, the lattice acts as a dissipation bath (thermal reservoir) that prevents a reversible transition between the metallic and insulating phases [9-19]. Moreover,

\footnotetext{
*Corresponding author: cedric.weber@kcl.ac.uk

†Corresponding author: shalaby@ swissterahertz.com
}

Published by the American Physical Society under the terms of the Creative Commons Attribution 4.0 International license. Further distribution of this work must maintain attribution to the author(s) and the published article's title, journal citation, and DOI. the nature of the MIT in $\mathrm{VO}_{2}$ has long been debated, with particular emphasis placed on the role of electron correlations in forming the charge gap [20-23]. A key unanswered question is whether the IMT is driven by a pure electronic mechanism, as in the Mott transition, or whether the vanadium dimer pairing mechanism driven by Peierls distortions is responsible for the opening of the charge gap [24-27]. This is paramount for future applications, such as low-consumption nonvolatile memory devices, and, on a more fundamental footing, to elucidate the nature of the transition in $\mathrm{VO}_{2}$.

In this work, we address the controversy on whether the IMT in $\mathrm{VO}_{2}$ is purely electronic or is driven by lattice distortions. We use two different routes: ultrafast terahertz $(\mathrm{THz})$ spectroscopy techniques and quasiparticle self-consistent $G W$ theory $(\mathrm{QS} G W)[28,29]$. While the two approaches, in our treatment, are independent, we reach the same conclusion.

We first describe the experimental restults. Structural properties are usually probed in equilibrium and for $\mathrm{VO}_{2}$ typically at temperatures near $T_{c}$. In our experiment, we use a $800-$ $\mathrm{nm}$ probe to investigate the ultrafast dynamics immediately following generation of phonons by a $\mathrm{THz}$ pump. Terahertz was generated using DSTMS/DAST organic crystals provided by [30]. In this way the dominant excitations well below $T_{c}$ can be observed. Our experimental techniques depend on THz-pump, optical probe spectroscopy (Fig. 1 and under Methods). The probe is a $800-\mathrm{nm}$ beam and detection is in two configurations: ellipsometry and transmission. Both can reveal electron densities and phonon oscillations. 
(a)

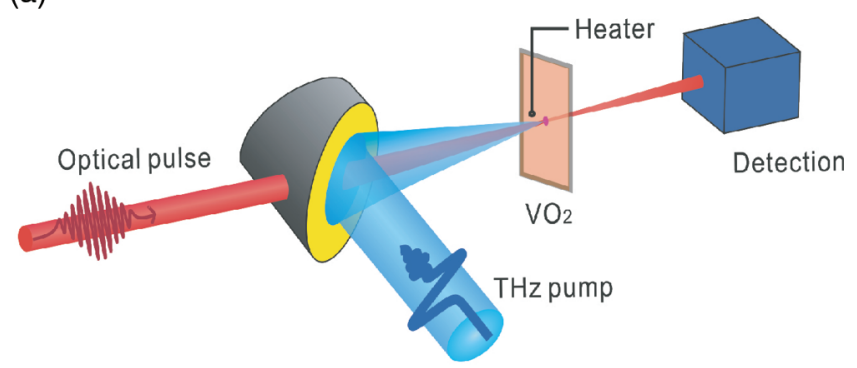

(b)

Ag- II

(c)
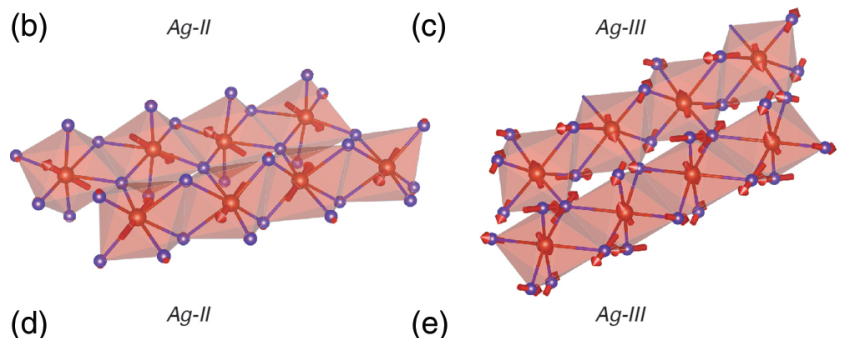

(e)

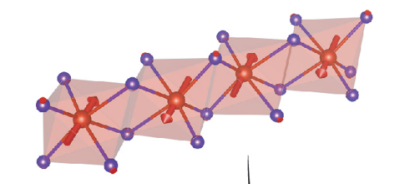

(f)

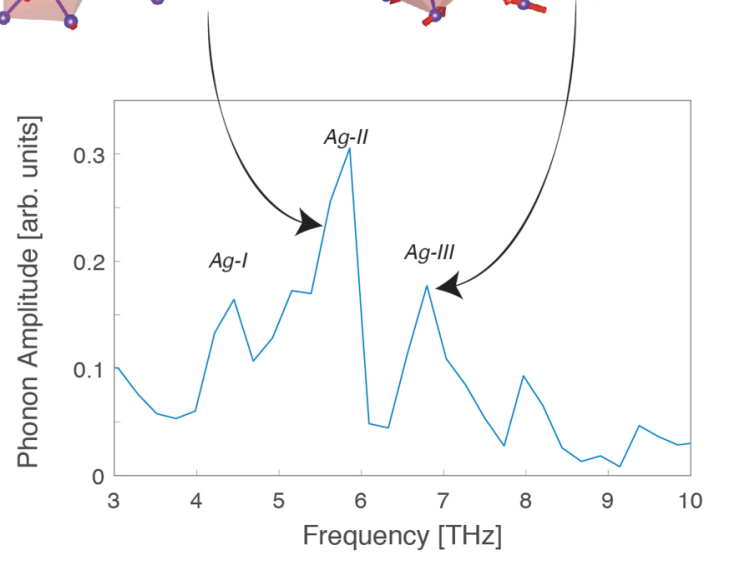

FIG. 1. The THz-induced lattice distortions far from $T_{c}$ in $\mathrm{VO}_{2}$. (a) The $\mathrm{THz}$ pump and optical probe setup. Lattice distortions obtained in the calculated phonon eigenvector modes for (b) the 5.7-THz A $\mathrm{A}_{g}$-II mode and (c) the 6.8-THz A -III mode. Panels (d) and (e) show the enlargement of panels (b) and (c), respectively, of the $\mathrm{V}$ (red spheres) and $\mathrm{O}$ (blue spheres) edge-sharing octahedras along the rutile direction. The $\mathrm{V}-\mathrm{V}$ dimers are located in the center of the rutile chain (second and third positions from the left). (f) The amplitude of phonon excitations measured by monitoring the THz-induced birefringence on a collinear $800-\mathrm{nm}$ probe.

However, ellipsometry is more sensitive to phonon oscillations and transmission is very sensitive to the electron density. Our sample is a 70 -nm-thick $\mathrm{VO}_{2}$ film on a sapphire substrate. High-quality polycrystalline $\mathrm{VO}_{2}$ thin films were grown on c-plane $\mathrm{Al}_{2} \mathrm{O}_{3}$ substrate by pulsed laser deposition. After the deposition, the sample was cooled down at constant oxygen pressure to room temperature.

In Fig. 2(a) we show the measured average temperaturetransmission hysteresis. As expected, a hysteresis in the (a)

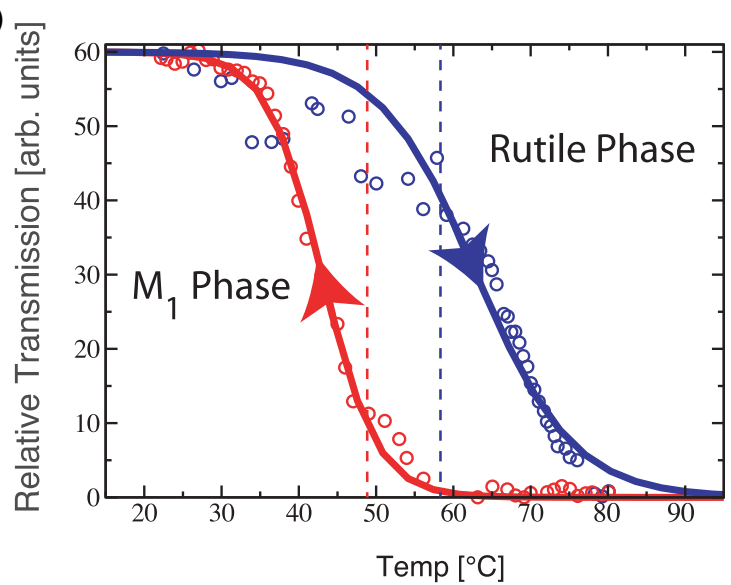

(b)
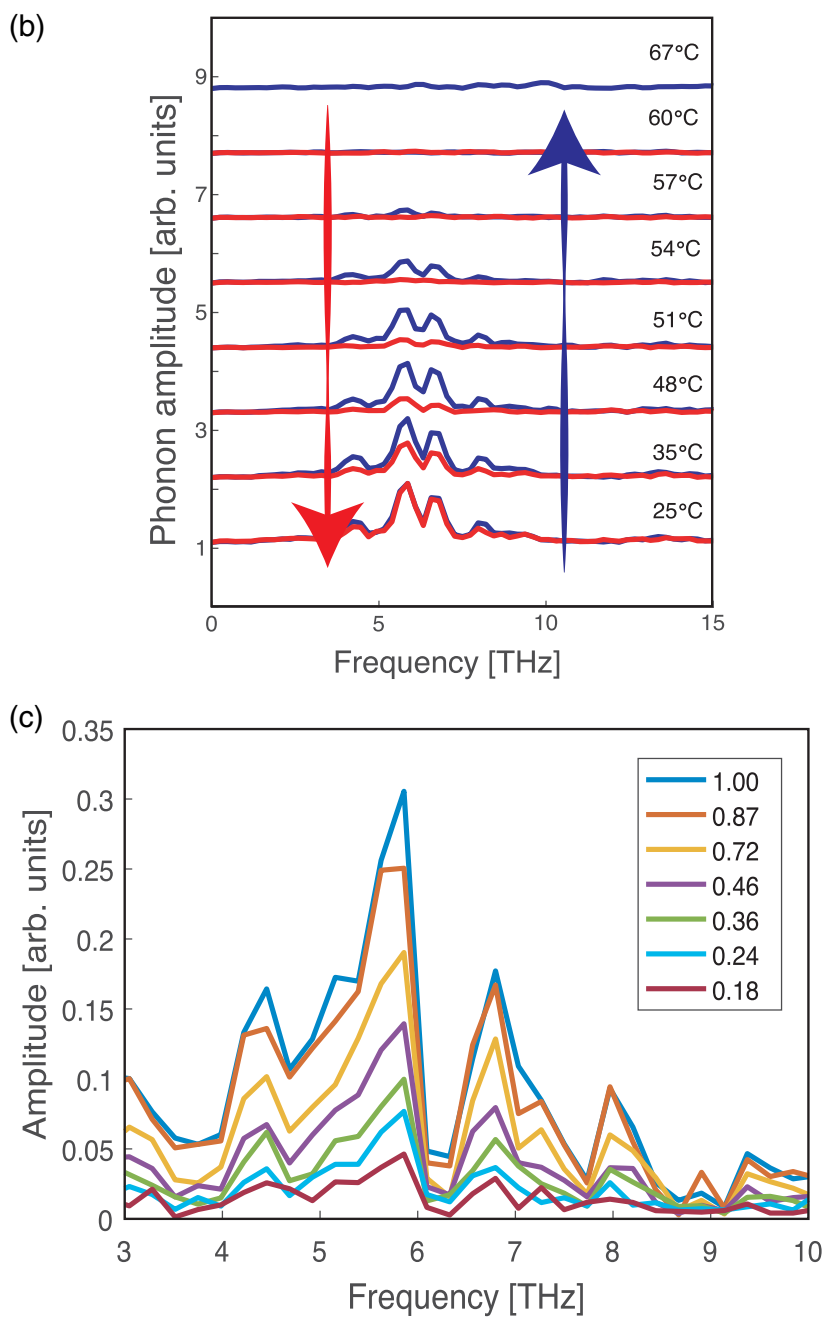

FIG. 2. Ultrafast THz spectroscopy of electron and phonon excitations. (a) Average transmission of the $800-\mathrm{nm}$ probe obtained during the heating (blue) and cooling experiments (red) and without $\mathrm{THz}$ excitation. (b) Electron-phonon dynamics obtained by Fourier transform of the time-resolved THz-pump optical ellipsometry measurements at different temperatures between $20^{\circ} \mathrm{C}$ up to $67^{\circ} \mathrm{C}$. Blue and red refer to the heating and cooling experiments and clearly show the hysteresis in dynamics (the hysteresis of the phonon modes follows the hysteresis of the structural properties, as expected). (c) The phonon spectra shown for different fluence levels of $\mathrm{THz}$ excitation at room temperature. 
transmission is observed, which correlates with the first-order transition of $\mathrm{VO}_{2}$ at $67^{\circ} \mathrm{C}$. However, the temporal response is highly nontrivial.

In order to track the phonon excitations, we first show the spectroscopic ellipsometry measurements (birefringence) [see Fig. 1(a)] carried out along the rutile axis with the $\mathrm{THz}$ excitation using the 800 -nm probe $(1.55 \mathrm{eV})$ for temperatures ranging between room temperature and the transition at $67^{\circ} \mathrm{C}$. We observed clear oscillations at room temperature with amplitude decreasing as the sample is heated up toward the transition (see Fig. 2S in the Supplemental Material [31]). The oscillations start simultaneously with the instantaneous electronic excitation (see Fig. 3S in the Supplemental Material [31]). To get better understanding on the nature of oscillations, we show the corresponding spectra in Fig. 2(b). For all temperatures below $57^{\circ} \mathrm{C}$, a coherent temporal response is observed, with three dominant modes at 4.8, 5.7, and 6.8 THz [see Fig. 2(b)]. These frequencies closely match known phonon modes in the $\mathrm{M}_{1}$ phase near $5 \mathrm{THz}$ (see Table 1 in the Supplemental Material [31]).

It is noteworthy to estimate the change of temperature induced by the $\mathrm{THz}$ pulse. In particular, the heat equation for the THz pulse is $Q=m C \Delta t$, where $m$ is the mass of the sample exposed to the beam, $C$ is the heat capacity, and $\Delta t$ is the obtained temperature variation. The heat capacity of $\mathrm{VO}_{2}$ thin films is typically [32] $C \approx 3 \mathrm{~J} \mathrm{~cm}^{-3} \mathrm{~K}^{-1}$. All fluences used in the experiment were below $1 \mathrm{~mW}$, with a sub-150- $\mu \mathrm{m}$ beam diameter, a sample thickness of $70 \mathrm{~nm}$, and a mass density of $\mathrm{VO}_{2}$ in monoclinic phase of $4.57 \mathrm{~g} \mathrm{~cm}^{-3}$ [33]. We obtain for an applied pulse of 2 ps a change of temperature $\Delta t \approx 0.001 \mathrm{~K}$, which is negligible in comparison to the monoclinic to rutile transition temperature.

Interestingly, the amplitude of these modes also exhibit hysteresis [see Fig. 2(b) and Fig. 2S]: The modes survive on heating to $57^{\circ} \mathrm{C}$ but only reappear on cooling from high temperature at $50^{\circ} \mathrm{C}$. The hysteresis of the excitations, however, correlates with the hysteresis obtained in the averaged transmission [see Fig. 2(a)], which shows that the excitations are an inherent property of the $\mathrm{M}_{1}$ phase of $\mathrm{VO}_{2}$. The fact that the excitations are present all the way to the phase transition establishes that the collective excitations are connected to the first-order transition of $\mathrm{VO}_{2}$ and the collapse of the $\mathrm{VO}_{2}$ gap at $T_{c}$.

We stress that the hysteresis of the phonon amplitude with temperature does not confirm the role of the lattice in the excitation. In principle, such hysteresis is expected regardless of the stimulus or the underlying mechanism behind the excitation. However, the ultrafast excitation (subpicosecond) of the oscillations, especially at room temperature far from transition (see Fig. 2S), strongly implies indispensable role of the phonon/lattice in the transition. This also suggest that the mechanism is Peierls-like, in the sense that changes in electronic states are driven by small nuclear displacements.

The excited phonon modes are Raman modes, and thus resonant excitation with the $\mathrm{THz}$ pulse is excluded. We performed fluence-dependent measurements in order to confirm the nature of the excitations. A fluence analysis [see Fig. 2(c) and Fig. 1S] shows the modes' amplitudes vary linearly with the square of the electric field. This establishes that modes are not resonantly excited by the $\mathrm{THz}$ pump but involve sec- ondary relaxation mechanisms associated with the coupling of electrons to active phonon modes. With THz spectroscopy, electronic relaxation does not need to obey optical selection rules but can occur through phonon coupling. We note that the dominant mode is at $5.7 \mathrm{THz}$.

For the sake of completeness, besides the birefringence/ phonon measurements shown above, we performed transmission measurements. As similar transmission studies have been previously studied, we include it in the Supplemental Material [31]. Moreover, in the study of phonon excitations, strong transmission changes makes it difficult to trace phonon oscillations. The conclusions reached above using experimental techniques can be confirmed using a different route of firstprinciples theoretical calculations as shown next.

\section{OPTICS}

To benchmark the QS $G W$ approximation, we compute the optical conductivity including ladder diagrams via a BetheSalpeter formalism [34]. We report in Fig. 3 the theoretical optical conductivity along the rutile axis and the comparison with ellipsometry measurement [35] of $\sigma_{x}$ on a single crystal of $\mathrm{VO}_{2}$. Agreement is excellent up to $4 \mathrm{eV}$, especially when considering the complexity of this material and also the variability between different measurements of $\sigma$. The optical conductivity is a stringent test of the quality of QS $G W$, as it is a true $a b$ initio theory, free of adjustable parameters.

\section{PHONONS}

We computed the phonon dynamical matrix at the densityfunctional-theory level (see Table 1 in the Supplemental Material [31]). The theoretical phonon spectrum exhibits three modes in the range 4.8 to $7 \mathrm{THz}$, all of $\mathrm{A}_{g}$ symmetry (denoted here as $\mathrm{A}_{g}-\mathrm{I}, \mathrm{A}_{g}-\mathrm{II}$, and $\left.\mathrm{A}_{g}-\mathrm{III}\right)$ [see Fig. 1(b)-1(e)]. The phonon frequencies are in excellent agreement with the modes observed by THz spectroscopy [see Fig. 1(f)], and the phonon eigenvectors supply the nuclear displacements for each normal mode. The $\mathrm{A}_{g}-\mathrm{II}\left(\mathrm{A}_{g}-\mathrm{I}\right)$ involves only displacements of the vanadium (oxygen) atoms, whereas the $\mathrm{A}_{g}$-III mode involves displacement of both $\mathrm{V}$ and $\mathrm{O}$ ions.

\section{A. Electron-phonon interaction}

We performed QS $G W$ calculations in a frozen phonon approximation, displacing atomic positions along eigenvectors of each of the three $\mathrm{A}_{g}$ modes $4.81,5.47$, and 6.29 THz. In two of these modes ( 4.8 and $6.29 \mathrm{THz})$, a twofold rotation around the $y$ axis is preserved, but it is broken for the $\mathrm{A}_{g}$-II mode (5.47 $\mathrm{THz}$, see Table 1 in the Supplemental Material [31]). Figure 4(a) shows how the quasiparticle band structure evolves for small displacements of either sign $(\delta d \approx \pm 0.022 \AA)$ in the $\mathrm{A}_{g}$-II mode. The nearly degenerate pair of $d$ bands just below $E_{F}$ split in a symmetric manner, independent of the sign of displacement - a characteristic signature of Peierls splitting — while the other bands are largely unperturbed. As a result the gap decreases in proportion to $|\delta d|$ until it closes at $\delta d \sim 0.14 \AA$ [Fig. 4(b)]. Note that a negative $\Delta_{c}$ means that the solution is metallic: The conduction band at $\Gamma$ overlaps with the valence band, with emergence of electron and hole pockets. 

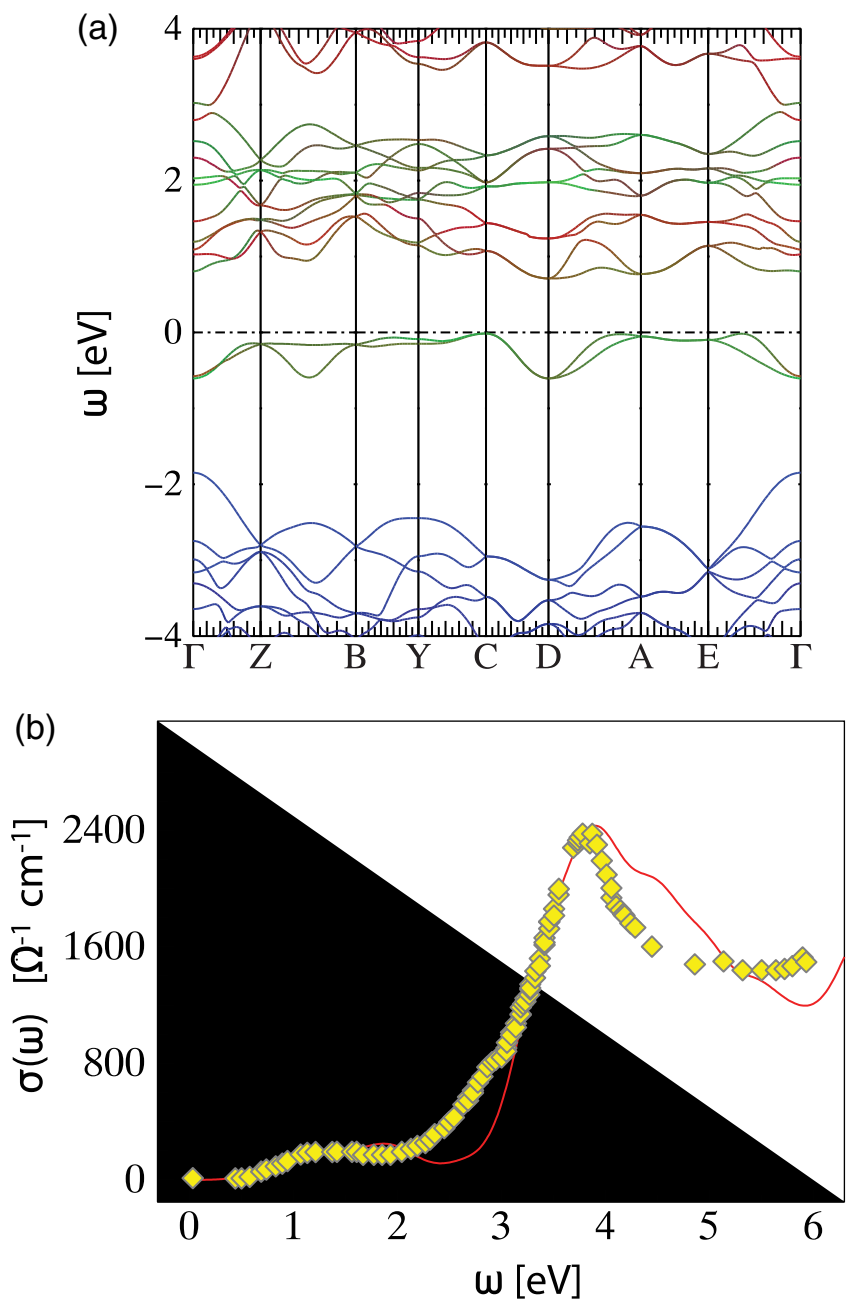

FIG. 3. Theoretical description of pristine $\mathrm{VO}_{2}$. (a) QS $G W$ band structure for pristine vanadium dioxide in the $\mathrm{M}_{1}$ phase in the absence of THz pumping . The charge gap is $0.67 \mathrm{eV}$. Orbital character is distinguished by a Mulliken projection onto the muffin-tin orbitals: O-p (blue), $\mathrm{Cu}-\mathrm{d} e_{g}$ (red), and $t_{2 g}$ (green). (b) Optical conductivity $\sigma_{x}$ (the rutile direction) obtained solving Bethe-Salpeter equation (QS $G W+\mathrm{BSE}$ ) for the polarizability starting from the QS $G W$ Hamiltonian. Agreement with ellipsometry data (diamonds) obtained in thin-film single crystal vanadium dioxide (in absence of $\mathrm{THz}$ pumping) [35] is remarkable for this notoriously difficult compound.

For comparison, a typical high power $\mathrm{THz}$ pump will displace ions by roughly $0.1 \AA$, while nearest-neighbor $\mathrm{V}-\mathrm{V}$ bond lengths in the rutile and $\mathrm{M}_{1}$ phase differ by $\sim 0.4 \AA$. In the $\mathrm{THz}$ experiment, the ions are displaced in a complex manner, so a realistic simulation of the time dependence of $\sigma(\omega=1.5 \mathrm{eV})$ is not feasible. However, the conductivity $\sigma(\omega)$ is closely connected to independent particle transitions (in particular the structure around $1.5 \mathrm{eV}$ is tied to transitions between the top two valence band states and the unoccupied states), so the initial shape of $\sigma(\omega)$ in the $\mathbf{M}_{1}$ phase (see Fig. $5 \mathrm{~S}(\mathrm{~b})$ in the Supplemental Material [31]) will evolve with excursions in the band structure [Fig. 1S(a)] from phonons generated by the pulse. This explains qualitatively the primary features of the $\mathrm{THz}$ experiment.
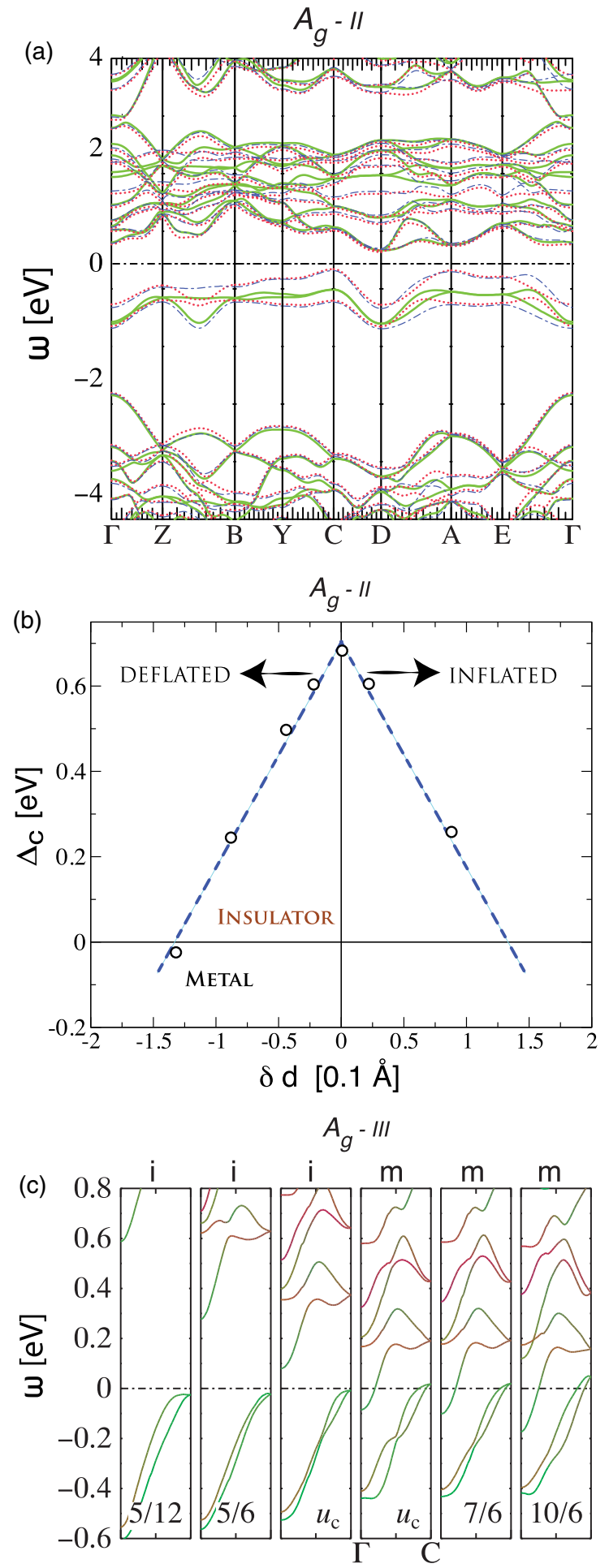

FIG. 4. Metal-insulating transition driven by lattice. (a) QS $G W$ energy-band calculations for the displaced structure along the $\mathrm{A}_{g}$-II (5.47 THz) phonon mode, with both expansions and contractions of the V-V bond ( $\delta d \approx \pm 0.022 \AA$ ) (red and blue) around the $\mathrm{M}_{1}$ phase (green). (b) The charge gap $\Delta_{c}$ shrinks for either sign of $\delta d$ and eventually closes for changes in the V-V distance as small as $\delta d \approx 0.15 \AA$. (c) QS $G W$ energy-band structure for the $\mathrm{A}_{g}$-III (e.g., $6.29 \mathrm{THz}$ ) phonon mode for displacements above and below the critical displacement $u_{c}$, see text. At $u_{c}$, QS $G W$ predicts the coexistence of two converged solutions: a metallic solution labeled $\mathbf{m}$ and an insulating solution labeled $\mathbf{i}$. 
We next turn to the discussion of the $\mathrm{A}_{g}$-III mode at 6.29 THz. For this particular mode, the valence band does not split and the charge gap evolves as a smooth function of phonon amplitude $u$, approaching a small positive value at a critical value we denote as $u_{c}$ [Fig. 4(c) and Fig. 6S(b)]. To provide some measure of the length scales involved, at $u_{c}$ the nuclei are displaced by an average value of $0.06 \AA$, though the nearest-neighbor V-V bond length increases by only $0.017 \AA$. As $u$ increases slightly beyond $u_{c}$ the gap vanishes; the insulating solution becomes unstable at $u_{c^{\prime}}>u_{c}$ and the system makes a discontinuous transition to a metallic state, with an indirect negative gap of the order of $-0.15 \mathrm{eV}$, meaning that the conduction band minimum (at $\Gamma$ ) falls slightly below the valence band maximum (which occurs at a low-symmetry point roughly in the vicinity of C). Figure 4(c) displays the evolution of the bands along the $\Gamma-\mathrm{C}$ line.

\section{THEORETICAL PREDICTION FOR BISTABILITY}

Remarkably, at $u_{c}$ two distinct self-consistent potentials can simultaneously be found for the same lattice configuration : one insulating and the other metallic. Both band structures are depicted in Fig. 4(c). This indicates that a purely electronic transition is possible in this system. The metal-to-insulator (M-I) transition here is very atypical, in that the associated fluctuations are purely chargelike, without involving the spin. Note that the band structures of the coexisting solutions are similar; however, there is a discontinuous change both in $\Delta_{c}$ and the valence band width. The narrowing of the bandwidth in the metallic solution indicates that the system is a correlated metal.

As a consequence of the coexistence of the two solutions, we obtain a hysteresis; with an excursion in the displacement $u_{c}<u<u_{c^{\prime}}$ the system can remain on one branch or the other. The subtle balance between the localized and itinerant character of the electrons in this regime is a realization of a spectral-weight scale : If the valence and conduction bands get slightly closer, then the system gets metallic; if they move apart, then the system becomes insulating. Note that the MI transition preserves the optical gap, as the charge gap is indirect. This is distinct from the insulator-metal transition in $\mathrm{VO}_{2}$ at the critical temperature, where there is a collapse of the optical gap concomitant with a collapse of the charge gap. We note that this process occurs on the timescale of a phonon mode oscillation period, which is typically much faster than the relaxation of resonant electronic excited modes.

\section{CONCLUSION}

We showed that a purely electronically driven transition does not occur for $\mathrm{VO}_{2}$ at the critical temperature. This conclusion is obtained by cross examination of experimental evidence that coherent phonon modes are activated below the transition and theoretical evidence that the latter modes are associated with a systematic reduction of the charge gap, and hence the buildup of metallicity. This concretely addresses a long-standing controversy on the role of phonons in the transition where the $\mathrm{M}_{1}$ phase of $\mathrm{VO}_{2}$ is a band insulator with a gap that is too large for pure many-body effects to stabilize a MIT without nuclear displacements.

Nevertheless, with suitable displacements relative to the $\mathrm{M}_{1}$ phase, a purely electronic M-I transition was found unveiling a new IMT mechanism: the Peierls instability, which involves an orbital selection and bonds the $d_{x y}$ and $d_{x z}$ orbitals along the rutile axis, filling each orbital with one electron. Nuclear displacements around the $\mathrm{M}_{1}$ phase causes the gap to shrink. When the gap becomes sufficiently small, a purely electronic MIT associated with the 6.8-THz phonon mode arises from the charge fluctuations.

This new theoretically predicted mechanism may enable bistability at room temperature in distorted structures which may be achieved by strain or external doping thus opening new possibilities for materials design under realistic experimental conditions.

\section{ACKNOWLEDGMENTS}

This work was supported by EPSRC (Grants No. EP/R02992X/1, No. EP/N02396X/1, No. EP/M011631/1) and the Simons Many-Electron Collaboration. C.W. gratefully acknowledges the support of NVIDIA Corporation with the donation of the Tesla K40 GPUs used for this research. For computational resources, we were supported by the ARCHER UK National Supercomputing Service and the UK Materials and Molecular Modelling Hub for computational resources (EPSRC Grant No. EP/ P020194/1). We acknowledge PRACE for awarding us access to SuperMUC at GCS@LRZ, Germany. The authors acknowledge the support of Swiss Terahertz GmbH with DAST/DSTMS organic crystals and RIGI THz camera for the experiment.
[1] F. J. Morin, Phys. Rev. Lett. 3, 34 (1959).

[2] I. Valmianski, P. Y. Wang, S. Wang, J. G. Ramirez, S. Guénon, and I. K. Schuller, Phys. Rev. B 98, 195144 (2018).

[3] D. Li, A. A. Sharma, D. K. Gala, N. Shukla, H. Paik, S. Datta, D. G. Schlom, J. A. Bain, and M. Skowronski, ACS Appl. Mater. Interfaces 8, 12908 (2016).

[4] E. Janod, J. Tranchant, B. Corraze, M. Querré, P. Stoliar, M. Rozenberg, T. Cren, D. Roditchev, V. T. Phuoc, M.-P. Besland, and L. Cario, Adv. Funct. Mater. 25, 6287 (2015).

[5] C. H. Ahn, J. M. Triscone, and J. Mannhart, Nature 424, 1015 (2003).
[6] Z. Yang, C. Ko, and S. Ramanathan, Annu. Rev. Mater. Res. 41, 337 (2011).

[7] M. Schultze, E. M. Bothschafter, A. Sommer, S. Holzner, W. Schweinberger, M. Fiess, M. Hofstetter, R. Kienberger, V. Apalkov, V. S. Yakovlev, M. I. Stockman, and F. Krausz, Nature 493, 75 (2012).

[8] S. de Jong, R. Kukreja, C. Trabant, N. Pontius, C. F. Chang, T. Kachel, M. Beye, F. Sorgenfrei, C. H. Back, B. Bräuer, W. F. Schlotter, J. J. Turner, O. Krupin, M. Doehler, D. Zhu, M. A. Hossain, A. O. Scherz, D. Fausti, F. Novelli, M. Esposito, W. S. Lee, Y. D. Chuang, D. H. Lu, R. G. Moore, M. Yi, M. Trigo, P. 
Kirchmann, L. Pathey, M. S. Golden, M. Buchholz, P. Metcalf, F. Parmigiani, W. Wurth, A. Föhlisch, C. Schüßler-Langeheine, and H. A. Dür, Nat. Mater. 12, 882 (2013).

[9] A. Cavalleri, C. Tóth, C. W. Siders, J. A. Squier, F. Ráksi, P. Forget, and J. C. Kieffer, Phys. Rev. Lett. 87, 237401 (2001).

[10] C. Kübler, H. Ehrke, R. Huber, R. Lopez, A. Halabica, R. F. Haglund, and A. Leitenstorfer, Phys. Rev. Lett. 99, 116401 (2007).

[11] A. X. Gray, M. C. Hoffmann, J. Jeong, N. P. Aetukuri, D. Zhu, H. Y. Hwang, N. C. Brandt, H. Wen, A. J. Sternbach, S. Bonetti, A. H. Reid, R. Kukreja, C. Graves, T. Wang, P. Granitzka, Z. Chen, D. J. Higley, T. Chase, E. Jal, E. Abreu, M. K. Liu, T.-C. Weng, D. Sokaras, D. Nordlund, M. Chollet, R. Alonso-Mori, H. Lemke, J. M. Glownia, M. Trigo, Y. Zhu, H. Ohldag, J. W. Freeland, M. G. Samant, J. Berakdar, R. D. Averitt, K. A. Nelson, S. S. P. Parkin, and H. A. Dürr, Phys. Rev. B 98, 045104 (2018).

[12] F. Zhang and C. L. Kane, Phys. Rev. B 90, 020501(R) (2014).

[13] G. Stefanovich, A. Pergament, and D. Stefanovich, J. Phys.: Condens. Matter 12, 8837 (2000).

[14] M. F. Jager, C. Ott, P. M. Kraus, C. J. Kaplan, W. Pouse, R. E. Marvel, R. F. Haglund, D. M. Neumark, and S. R. Leone, Proc. Natl. Acad. Sci. USA 114, 9558 (2017).

[15] G. Gopalakrishnan, D. Ruzmetov, and S. Ramanathan, J. Mater. Sci. 44, 5345 (2009).

[16] B. Simon Mun, J. Yoon, S.-K. Mo, K. Chen, N. Tamura, C. Dejoie, M. Kunz, Z. Liu, C. Park, K. Moon, and H. Ju, Appl. Phys. Lett. 103, 061902 (2013).

[17] S. Guénon, S. Scharinger, S. Wang, J. G. Ramírez, D. Koelle, R. Kleiner, and I. K. Schuller, Europhys. Lett. 101, 57003 (2013).

[18] G. M. Liao, S. Chen, L. L. Fan, Y. L. Chen, X. Q. Wang, H. Ren, Z. M. Zhang, and C. W. Zou, AIP Adv. 6, 045014 (2016).

[19] B. A. Kruger, A. Joushaghani, and J. K. S. Poon, Opt. Express 20, 23598 (2012).
[20] J. M. Tomczak, F. Aryasetiawan, and S. Biermann, Phys. Rev. B 78, 115103 (2008).

[21] R. Sakuma, T. Miyake, and F. Aryasetiawan, J. Phys.: Condens. Matter 21, 064226 (2009).

[22] S. Biermann, A. Poteryaev, A. I. Lichtenstein, and A. Georges, Phys. Rev. Lett. 94, 026404 (2005).

[23] W. H. Brito, M. C. O. Aguiar, K. Haule, and G. Kotliar, Phys. Rev. Lett. 117, 056402 (2016).

[24] M. M. Qazilbash, A. A. Schafgans, K. S. Burch, S. J. Yun, B. G. Chae, B. J. Kim, H. T. Kim, and D. N. Basov, Phys. Rev. B 77, 115121 (2008).

[25] J. H. Park, J. M. Coy, T. S. Kasirga, C. Huang, Z. Fei, S. Hunter, and D. H. Cobden, Nature 500, 431 (2013).

[26] N. B. Aetukuri, A. X. Gray, M. Drouard, M. Cossale, L. Gao, A. H. Reid, R. Kukreja, H. Ohldag, C. A. Jenkins, E. Arenholz, K. P. Roche, H. A. Dürr, M. G. Samant, and S. S. P. Parkin, Nat. Phys. 9, 661 (2013).

[27] S. Cueff, D. Li, Y. Zhou, F. J. Wong, J. A. Kurvits, S. Ramanathan, and R. Zia, Nat. Commun. 6, 8636 (2015).

[28] M. van Schilfgaarde, T. Kotani, and S. Faleev, Phys. Rev. Lett. 96, 226402 (2006)

[29] D. Pashov, S. Acharya, W. R. L. Lambrecht, J. Jackson, K. D. Belashchenko, A. Chantis, F. Jamet, and M. van Schilfgaarde, Comput. Phys. Commun. 249, 107065 (2020).

[30] Swiss Terahertz GmbH.

[31] See Supplemental Material at http://link.aps.org/supplemental/ 10.1103/PhysRevResearch.2.023076 for additional theoretical and experimental data.

[32] D.-W. Oh, C. Ko, S. Ramanathan, and D. G. Cahill, Appl. Phys. Lett. 96, 151906 (2010).

[33] H. Wen, L. Guo, E. Barnes, J. H. Lee, D. A. Walko, R. D. Schaller, J. A. Moyer, R. Misra, Y. Li, E. M. Dufresne, D. G. Schlom, V. Gopalan, and J. W. Freeland, Phys. Rev. B 88, 165424 (2013).

[34] B. Cunningham, M. Grüning, P. Azarhoosh, D. Pashov, and M. van Schilfgaarde, Phys. Rev. Mater. 2, 034603 (2018).

[35] K. Okazaki, S. Sugai, Y. Muraoka, and Z. Hiroi, Phys. Rev. B 73, 165116 (2006). 